\title{
Newton Did Not Invent or Use the So-Called Newton's Gravitational Constant; $G$, It Has Mainly Caused Confusion
}

\author{
Espen Gaarder Haug \\ Norwegian University of Life Sciences, Ås, Norway \\ Email: espenhaug@mac.com
}

How to cite this paper: Haug, E.G. (2022) Newton Did Not Invent or Use the SoCalled Newton's Gravitational Constant; $G$, It Has Mainly Caused Confusion. Journal of Modern Physics, 13, 179-205.

https://doi.org/10.4236/jmp.2022.132014

Received: December 11, 2021

Accepted: February 19, 2022

Published: February 22, 2022

Copyright $\odot 2022$ by author(s) and Scientific Research Publishing Inc. This work is licensed under the Creative Commons Attribution International License (CC BY 4.0).

http://creativecommons.org/licenses/by/4.0/

\begin{abstract}
Newton did not invent or use the so-called Newton's gravitational constant $G$. Newton's original gravity formula was $F=\frac{M m}{R^{2}}$ and not $F=G \frac{M m}{R^{2}}$. In this paper, we will show how a series of major gravity phenomena can be calculated and predicted without the gravitational constant. This is, to some degree, well known, at least for those that have studied a significant amount of the older literature on gravity. However, to understand gravity at a deeper level, still without $G$, one needs to trust Newton's formula. It is when we first combine Newton's assumptionn, that matter and light ultimately consist of hard indivisible particles, with new insight in atomism that we can truly begin to understand gravity at a deeper level. This leads to a quantum gravity theory that is unified with quantum mechanics and in which there is no need for $G$ and not even a need for the Planck constant. We claim that two mistakes have been made in physics, which have held back progress towards a unified quantum gravity theory. First, it has been common practice to consider Newton's gravitational constant as almost holy and untouchable. Thus, we have neglected to see an important aspect of mass; namely, the indivisible particle that Newton also held in high regard. Second, standard physics have built their quantum mechanics around the de Broglie wavelength, rather than the Compton wavelength. We claim the de Broglie wavelength is merely a mathematical derivative of the Compton wavelength, the true matter wavelength.
\end{abstract}

\section{Keywords}

Newton Gravity, Newton's Gravitational Constant, Schwarzschild Radius, Quantum Gravity, Planck Length 


\section{Newton Neither Invented Nor Used $G$}

In his book, the Principia [1], Newton mentioned the gravitational force formula in words (see the Appendix) that create an equivalent to:

$$
F=\frac{M m}{R^{2}}
$$

However, he did not make a single mention of any gravitational constant (with the notation of $G$ or through any other notation), nor did he ever use such a constant himself. This appears to be something that few physicists or historians today know or acknowledge [2] [3]. In the Principia, Newton's focus was on relative masses, although he mentioned the word "mass" only once, but it is clear that he meant mass as an amount of matter. Based on easily-observable gravitational observations, such as the orbital time of satellites (moons and planets), he found the relative mass (weight) of Saturn, Jupiter, the Earth, and the sun; see also Cohen [4] for more detail on this. Cohen also pointed out that Newton's focus is on relative masses:

"That is, since Newton is concerned with relative masses and relative densities, the test mass can take any unity"

The kilogram definition of mass was invented more than 100 years after Newton published the Principia and thus came into being long after his death. Newton was, in several of his texts, clear on the idea that matter (and energy) at the deepest level is based on indivisible fully-hard particles with spatial dimension. He took this idea from atomism, a source that he referred to several times in his work [5] [6]. Newton was focused on atomism before he started to publish his work; this is evident from his unpublished notebook. He was also clear on this in Principia and, in particular, in his later book Opticks [7]. Newton thought that the amount of mass was related to the quantity of indivisible particles in the chosen mass. He even assumed that light was made up of such indivisible particles. He knew that it was impossible to find the number of indivisible particles in any observable mass at that time, an assertion that he mentioned in Principia. It was therefore natural for him to focus on relative masses when he worked with gravity. In short, to find the relative mass of two heavenly objects, Newton utilized satellite orbital time and the distance from the satellite to the center of the mass of which he wanted to find the relative mass; this is a method we return to shortly.

Newton also explained that weight is proportional to mass. In other words, twice the mass gives twice the weight in relation to two masses located the same distance from the gravitational object.

In 1798, Henry Cavendish [8] measured the density of the Earth using a torsion balance, also known as a Cavendish apparatus. The principles of such apparatus was already described by geologist John Michel [9] in 1784, but he died before he was able to use it, and Cavendish gives him full credit for the idea. Earlier, Newton had found the relative density between planets, and for this no Cavendish apparatus or similar was needed. However, when we want to find the 
density of the Earth relative to a given substance, for example, water or iron, we need to know the gravity properties of a mass that we know is formed uniformly of the chosen substance. The Cavendish apparatus was needed to measure the gravitational effect from a small practical mass when one had knowledge of what substance it contained. Based on knowing the gravitational effect from such a known substance, one could compare that to the gravitational effect of the Earth and then know the density of the Earth relative to this substance.

Cavendish did not mention a gravitational constant nor did he have use for one. However, a Cavendish apparatus can indeed be used to find the gravitational constant $G$. The gravitational constant was needed when one decided on the kilogram definition of mass. Even if the kg definition was likely already introduced in 1796, it did not become widely used before around the 1870s. An important change here is that the Metre Convention was signed in 1875, leading to production of The International Prototype of the Kilogram. The kg definition of mass is, in our view, an incomplete definition of mass that needs $G$ to become a complete mass measure that incorporates gravity effects from matter. The gravitational constant was likely mentioned for the first time by the French physicists Cornu and Baille [10] in 1873. Their paper mentioned the gravity force formula in the form $F=f m^{\prime} / R^{2}$, where $f$ is the gravitational constant.

However, the idea took hold and in 1894, the gravitational constant was first called $G$ (rather than $f)$ by Boys [11] in a proceeding at the Royal Society that followed shortly after he published in the prestigious journal Nature. To switch the notation from $f$ to $G$ is simply cosmetic ${ }^{1}$. Although, for example, Max Planck still used the notation $f$ for the gravity constant in 1899, 1906, and 1928 [12] [13] [14], the use of $G$ continued, and by the 1930 s $G$ had become the standard notation for the gravitational constant. Keep in mind that it took 200 years from the publication of Newton's gravitational theory to the first mention of the gravitational constant; thus it was, to some degree, a breakthrough, but from another perspective, it could also be seen as a disaster, as it led to an inferior definition of mass.

2. Newton's Gravity Formula; $F=\frac{\tilde{M} \tilde{m}}{R^{2}}$

As the original Newton formula is not compatible with the kg definition of mass (without adding a gravitational constant), we will call the Newton mass $\tilde{M}$ to distinguish it from the modern $\mathrm{kg}$ definition of mass $M$. We will later explain why the mass we obtain from the original Newton formula is superior to the $\mathrm{kg}$ definition of mass.

The centripetal force in the Newtonian theory is given by $\frac{\tilde{m} v^{2}}{R}$. For a planet or moon to be in equilibrium within its orbit, the centripetal force must balance

${ }^{1}$ But Boys also had some interesting information in his paper on measurement methods in relation to $G$, for example. 
with the gravitational force, so under the original Newton theory we must have:

$$
\frac{\tilde{m} v^{2}}{R}-\frac{\tilde{M} \tilde{m}}{R^{2}}=0
$$

Solved with respect to $v$, this gives an orbital velocity of:

$$
v=\sqrt{\frac{\tilde{M}}{R}}
$$

As we can see, this is quite different from the modern orbital velocity formula that is $v=\sqrt{\frac{G M}{R}}$. The difference is the Newton gravitational constant $G$, which, as we have noted, Newton himself never used. We can then ask, "Does the formula work without the Newton gravitational constant?" And, in fact, it does. Newton used the square of the orbital time and the distance between two masses to find the relative masses of heavenly objects. The orbital time is the circumference of the orbiting object (for example the moon) divided by the orbital velocity. In other words:

$$
\begin{aligned}
& \frac{L}{v}=\frac{L}{\sqrt{\frac{\tilde{M}}{R}}} \\
& T=\frac{L}{\sqrt{\frac{\tilde{M}}{R}}}
\end{aligned}
$$

This formula we can then solve with respect to mass, and we get:

$$
\begin{gathered}
\tilde{M}=\frac{L^{2} R}{T^{2}} \\
\tilde{M}=\frac{(2 \pi R)^{2} R}{T^{2}} \\
\tilde{M}=\frac{4 \pi^{2} R^{3}}{T^{2}}
\end{gathered}
$$

Assume we decide to measure orbital time in days (as Newton did) and distance in $\mathrm{km}$ (although naturally Newton used a different length measure). The distance to the sun can be found by parallax, and it is about 149.6 million $\mathrm{km}$. The time it takes for the Earth to orbit the sun is 365 days. So now we can calculate the mass of the Sun as:

$$
\tilde{M}_{s}=\frac{4 \pi^{2} 149600000^{3}}{365^{2}} \approx 9.92 \times 10^{20} \mathrm{~km}^{3} / \text { days }^{2}
$$

As we can see, the mass has very strange notation and does not seem to be very recognizable or intuitive, but this is partly because we are accustomed to thinking of mass in terms of $\mathrm{kg}$ (or pounds). Next, let us calculate the mass of the Earth; for this we will use the orbital time of the moon, which is about 27.3 days. The distance from the Earth to the moon is about $384,400 \mathrm{~km}$. The mass of the Earth must therefore be: 


$$
\tilde{M}_{E}=\frac{4 \pi^{2} 384400^{3}}{27.3^{2}} \approx 3 \times 10^{15} \mathrm{~km}^{3} / \text { days }^{2}
$$

Again, this seems to be a strange mass that is hard for us relate to, but the mass of the sun relative to the Earth is now $\frac{9.92 \times 10^{20}}{3 \times 10^{15}} \approx 329750$. This is a number many of us do recognize; it is the mass of the sun relative to the Earth that we also obtain if we look at the modern kg definitions of the sun and the Earth. The $4 \pi^{2}$ will even cancel out in the relative mass formula, which can be described by:

$$
\frac{R_{1}^{3} T_{2}^{2}}{R_{2}^{3} T_{1}^{2}}
$$

Further, if the satellites were orbiting the objects we wanted to find the mass of at the same distance $r_{1}=r_{2}$, then the relative mass is simply the orbital time squared divided by each other. This is very similar to Newton's reasoning in the Principia. As Newton pointed out, one could use any units one wanted (for distance or time) when the focus was on relative masses. When we say the sun's mass is 329,750 times that of the Earth's, then we have chosen the Earth as the unit mass. We could just as well have used the Earth mass as the unit mass when handling small objects on Earth. However, the mass of the Earth is enormous compared to any object we handle in our daily lives and so it would be hard to conceptualize it. Therefore, to have a better understanding of the mass, it makes sense to choose a smaller unit mass. The $\mathrm{kg}$ is a unit mass that is an arbitrarily-chosen mass, but it is practical-not so small so that it was hard to measure on an old-fashioned scale, and yet not so big that it could not be carried around. Weights, we must remember, were important to standardized trade, for example. So, we can say an almost arbitrary amount of weight (mass) was chosen as a $\mathrm{kg}$. When we deal with a small practical mass, we can also quite easily know what substance it consists of-we can make a lead ball, gold ball, or iron ball, or we can simply fill a container with water. When we deal with planets, we know they likely consist of many types of elements, and it is harder to say for certain what their cores consist of completely.

Now to find the mass of the Earth in $\mathrm{kg}$, we must first find a method to test gravity's effect on small practical masses, e.g., where we already know the $\mathrm{kg}$ mass of the object in question. Remember that to find the mass of the sun, Newton needed something orbiting the sun, but obviously there are plenty of planets to choose from. To find the mass of the Earth, he needed something that orbited the Earth, and indeed, the moon fit the bill. However, in order to measure a small practical mass, we need something "orbiting"2 that is also very small (very small compared to planets, but still massive compared to atoms and molecules). This was a difficult task, and many attempts were undertaken, but it was first done accurately in 1798 by Henry Cavendish through what is known today as a "Cavendish apparatus" and consists of some small balls (made of lead or gold, 
for example) "orbiting" some larger (but still small) balls. Interestingly, the mass of a large lead ball in the Cavendish apparatus will have a Newton mass of:

$$
\tilde{M}=\frac{2 \pi^{2} L R^{2} \theta}{T^{2}}
$$

where $T$ is the oscillation time, and $\theta$ is the deflection angle of the torsion balance from its rest position, and $R$ is the distance from the small lead ball to the large lead ball, and $L$ is the distance between the two small balls.

We know how to find this Newtonian type mass with the torsion balance, Formula (7). We do not need to know its kg mass or any other mass-measure for this. However, we can find its $\mathrm{kg}$ mass by comparing it with the $\mathrm{kg}$ standard by using a scale calibrated to $\mathrm{kg}$. This now gives us a connection between the mysterious Newton mass and the $\mathrm{kg}$ (or pound). We can now also find the kg mass of the Earth, and the density of the Earth in terms of kg. The Cavendish apparatus, which was said to first find the gravitational constant indirectly, is both true and not true. Cavendish never mentioned a gravitational constant, and it is not needed under any circumstances, as we soon will see. The reason the Cavendish apparatus was required then was because one needed a way to measure the Newtonian type mass of a small object, so one could use the small unit (instead of the Earth, for example) as unit mass. The Cavendish apparatus also made it possible to accurately find the density of the Earth, not because of any gravitational constant, but because a small practical mass can be made of one substance where the density (weight) is known relative to other substances (e.g., gold versus water). In this way, one could find the density of the Earth very accurately relative to a given substance. If one had known a planet in our solar system consisted of a homogenous substance, take iron, for example, then there would have been no need for a Cavendish apparatus to find the density of the Earth relative to material objects. But we know of no such planet consisting of only one substance, and it would also be hard to check if that was really the case, even if it could be imagined. So, the breakthrough of the Cavendish apparatus was that one could find the gravity (Newtonian mass) of even a small practical mass. Naturally we can find the relative densities of different substances simply by using a scale.

Still, what we call the Newtonian mass, $\tilde{M}$, is difficult to fully understand, although it is no stranger than the $\mathrm{kg}$. Up until now, we have used arbitrary units such as $\mathrm{km}$ for length, and Earth days as time. As we will see, it is when we first switch to more fundamental units and then explore the quantum world that we truly see the beauty of Newton's formula.

\section{Switching to more fundamental units}

At this stage we can still choose any time unit we want: years, days, hours, or seconds. More important than the choice of time interval (time unit) is to link both time and length to something very fundamental in nature. This is light. We know from the writings of Aristotle (in his work De Sensu) that the Greek philosopher Empedocles, about $2500 \mathrm{BC}$, understood or at least assumed that the speed of light had a finite limit: 
Empedocles said that the light from the sun arrives first in the intervening space before it comes to the eye, or reaches the Earth. This might seem to be the case. For whatever is moved through space is moved from one place to another, hence, there must be a corresponding interval of time in which it is also moved from one place to the other.

In 1676, Ole Christensen Rømer was likely the first to make a quantitative measurement of the speed of light and he concluded that it was finite. In 1704, in his book Opticks [7], Newton reported Rømer's calculations of the finite speed of light and gave a value of "seven or eight minutes" for the time it would take for light to travel from the sun to the Earth, an estimate that is not far from its real speed. So, Newton could have linked length to time through the speed of light, even if his calculations and predictions would have been somewhat inaccurate. In 1728, (one year after Newton's death) the English physicist James Bradley estimated the speed of light in a vacuum to be approximately 301,000 $\mathrm{km}$ per second, which is very close to today's defined value.

Here we will choose seconds as the time unit, and will link this to length through the speed of light. Our length unit will be the distance light travels in any given time unit. Here we choose the second; this is a well-known unit distance in modern physics, known as light-second (length); see, for example [15]. Now time and length units are suddenly related to something very fundamental. In modern physics, the speed of light is the same in every reference frame; it is known as $c$ and per definition exactly 299,792,458 meters per second in vacuum. But here we have chosen the length unit that represents how long light travels in one second, so the speed of light will then be one light-second per second in this unit system. In other words, we can set $c=1$, something that is often done in modern physics. What is important is that time and length are linked through something very fundamental, namely the speed of light.

Now the distance to from the Earth to the sun will be about $R=149600000000 \mathrm{~m} / 299792458 \mathrm{~m} / \mathrm{s} \approx 499$ light-seconds. The circumference of the orbit of the Earth around the sun is therefore about $L=2 \pi \times 499$ light seconds. Further, we can find the mass of the sun

$$
\tilde{M}_{S}=\frac{4 \pi^{2} R^{3}}{T^{2}}=\frac{4 \pi^{2} 499^{3}}{(365 \times 24 \times 60 \times 60)^{2}} \approx 4.93 \times 10^{-6} \text { Light-seconds }
$$

This looks like a very unfamiliar mass, but soon we will see it makes much more sense than expressing the mass of the sun in $\mathrm{kg}$. (The sun's mass in $\mathrm{kg}$ is approximately $1.98 \times 10^{30}$ ).

Similarly, for the Earth we can use the moon's orbital time to find the mass of the Earth. The orbital time of the moon is about 27 days, or $27 \times 24 \times 60 \times 60$ seconds. The distance to the moon is about 1.28 light-seconds. The mass of the Earth must therefore be:

$$
\tilde{M}_{E}=\frac{4 \pi^{2} 1.28^{3}}{(27 \times 24 \times 60 \times 60)^{2}} \approx 1.52 \times 10^{-11} \text { Light-seconds }
$$

This means the mass of the sun relative to the Earth must be approximately 
$\frac{1.52 \times 10^{-11}}{4.93 \times 10^{-6}} \approx 324342$. This is close to the actual modern accepted number.

Next let us use the orbital velocity formula $v=\sqrt{\frac{\tilde{M}}{R}}$ to predict the orbital velocity of Saturn. The distance from the sun to Saturn is about 1.434 billion $\mathrm{km}$, which is about 4783.3 light-seconds. The mass of the sun we have estimated to be $4.93 \times 10^{-6}$ light seconds, and inputting the formula, we get:

$$
v=\sqrt{\frac{4.93 \times 10^{-6}}{4783.3}} \approx 3.21 \times 10^{-05} \text { Light-seconds per second }
$$

That is, the orbital velocity is now on the dimensionless form; it is identical to $\frac{v}{c}$. In order to obtain meters per second, we need to multiply by $c$ and this gives us about 9625 meters per second, which is the same as is observed in experiments. That our orbital velocity can actually be seen as $\frac{v}{C}$ means it is a dimensionless number. For example, Langacker [15] in his book "Can the Laws of Physics Be Unified?" (2017) indicated that such dimensionless units as $\frac{v}{C}$ could be more fundamental.

Actually, the mass we find in this way without depending on or knowing $G$ is identical to half the Schwarzschild radius in meters divided by the speed of light, and exactly equal to the Haug radius [16]; $r_{h}=\frac{G M}{R}=\frac{1}{2} r_{s}$, which is derived by taking into account relativistic mass that has been abandoned by general relativity theory [17] [18]. In other words, this is half the Schwarzschild radius in light-seconds or exactly the Haug radius in light-seconds. We propose that the Haug radius (divided by the speed of light) could be a much better model of mass than the kg-defined mass. However, no one should be fully convinced that light seconds are a better mass measure than $\mathrm{kg}$ just yet. It is when we get to the quantum aspects that this first becomes clear. As explained previously, we have demonstrated that we can predict relative masses, we can find the density of planets, and we can perform orbital velocity predictions, all with no knowledge of the gravitational constant. We will expand further on this before returning to look at the light-second mass from a quantum perspective.

\section{Escape Velocity and Such Things as Time Dilation}

Leibniz [19] already suggested the in 1688 that kinetic energy was given by $m v^{2}$ a formula that "soon" was empirically confirmed by Gravesande [20] around 1720. We know today this should be corrected to $E_{k} \approx \frac{1}{2} m v^{2}$ (ignoring relativistic effects, so valid for when $v \ll c$ ). The escape velocity in Newton's formula can be derived in the following way:

$$
\frac{1}{2} \tilde{m} v_{e}^{2}-\frac{\tilde{M} \tilde{m}}{R^{2}}
$$


and when we solve with respect to $v_{e}$, this gives

$$
v_{e} \approx \sqrt{\frac{2 \tilde{M}}{R}}
$$

We can also find expected gravitational time dilation by taking into account that the time of a clock at distance $R_{2}$ must move faster than the clock at a distance of $R_{1} \quad\left(R_{2}=R_{1}+h\right.$, where $h$ is the hight about ocean level) from the center of the gravity object by:

$$
\begin{gathered}
\frac{T_{2}}{\sqrt{1-v_{2}^{2}}}=\frac{T_{1}}{\sqrt{1-v_{1}^{2}}} \\
\frac{T_{2}}{\sqrt{1-\frac{2 \tilde{M}}{R_{2}}}}=\frac{T_{1}}{\sqrt{1-\frac{2 \tilde{M}}{R_{1}}}} \\
T_{2}=T_{1} \frac{\sqrt{1-\frac{2 \tilde{M}}{R_{2}}}}{\sqrt{1-\frac{2 \tilde{M}}{R_{1}}}}
\end{gathered}
$$

Assume the clock $T_{1}$ is at sea level and clock $T_{2}$ is 2,000 meters above sea level, which corresponds to $r_{1} \approx 6371000 / c=0.0212514$ light-seconds and $r_{2}=(6371000+2000) / c=0.0212580$ light-seconds. For every second at the ocean level, following number of seconds will go by as observed from the mountain level:

$$
T_{2}=1 \frac{\sqrt{1-\frac{2 \times 1.52 \times 10^{-11}}{0.0212580}}}{\sqrt{1-\frac{2 \times 1.52 \times 10^{-11}}{0.0212514}}}=1.00000000000022 \mathrm{~s}
$$

which is the same as predicted by general relativity theory. The point is that here we have done it without any knowledge of $G$. What is even more important is our mass. The mass of the Earth, as we have said, is about $1.52 \times 10^{-11}$ light-seconds. We can convert this to meters by multiplying by $c=299792458 \mathrm{~m} / \mathrm{s}$. This means the mass of the Earth is $1.52 \times 10^{-11} \times c=0.0046 \mathrm{~m}$. This is actually half of the Schwarzschild radius of the Earth and identical to the Haug radius, which is no coincidence. From Newton's formula, one finds that the mass is the Haug radius of the Earth (when using length units linked to how far light travels in the arbitrary chosen time unit, here seconds). One gets the Haug radius by $r_{h}=\frac{G M}{c^{2}}$; however, modern physics has not recognized that half the Schwarzschild radius actually is a better definition of mass than the kilogram mass, but a new quantum gravity theory has taken advantage of this [21] [22]. Be aware that Michell [9] already, in 1784, got exactly the same radius for where the escape velocity was $c$ as the much later Schwarzschild radius rooted in general relativity theory. So the Schwarzschild radius is not unique for general relativity theory [23]; they are the same. 


\section{Getting Down to the Quantum Level}

Any rest-mass in terms of $\mathrm{kg}$ can be expressed as:

$$
m=\frac{\hbar}{\bar{\lambda}} \frac{1}{c}
$$

where $\hbar$ is the Planck constant, $\bar{\lambda}$ is the reduced Compton length [24], and $c$ is the well-known speed of light. This formula ${ }^{3}$ can describe any rest-mass in terms of $\mathrm{kg}$, including both subatomic and cosmological objects. The Planck constant is indeed a constant, and so is the speed of light. The only factor that differs between masses of different sizes (weights) is then the Compton wavelength of the mass. The Compton wavelength has only been measured for fundamental particles such as the electron. However, even larger masses that don't have their own Compton wavelengths still consist of a series of subatomic particles that must have Compton wavelengths. The Compton wavelengths of elementary particles are additive based on the following formula:

$$
\bar{\lambda}=\sum_{i=1}^{n}=\frac{1}{\frac{1}{\bar{\lambda}_{1}}+\frac{1}{\bar{\lambda}_{2}}+\frac{1}{\bar{\lambda}_{3}}+\cdots+\frac{1}{\bar{\lambda}_{n}}}
$$

This means that the Formula (14) can be used for composite masses and even astronomical objects like the sun or the moon. But what does the formula truly represent? The Planck constant is linked to the quantization of energy. Some will find it strange that the speed of light is embedded in the mass formula. We are all familiar with $E=m c^{2}$, but few physicists are familiar with the idea that the speed of light is integrated in the mass at a deeper level. This indicates something inside a fundamental particle, a mass, is linked to the speed of light, and also to composite masses, as they consist of fundamental particles. But how? Mass is known at the quantum level to be a wave-particle duality. But what exactly is a wave-particle duality? Newton assumed light consisted of indivisible particles; later, the view that light was a wave evolved from some experiments strongly indicating wave behaviour. Then Einstein introduced his photoelectric effect and again showed that light had particle-like properties, and light was redefined as having a mystical wave-particle duality; not mystical in the terms of the math, but in terms of the interpretation of the math. Then Louis de Broglie [28] [29] suggested that matter, in addition to having particle-like properties, also likely had wave-like properties, and he suggested that the matter wave was given by the following formula $\lambda_{B}=\frac{\hbar}{m v \gamma}$, where $\gamma=\frac{1}{\sqrt{1-\frac{v^{2}}{c^{2}}}}$. Einstein quickly endorsed the idea, and some years later it was confirmed that masses such as electrons had wave-like properties; see [30] [31]. This was considered almost a ${ }^{3}$ This way of to describe the kilogram mass was possibly first described by Haug [25] [26] [27]. To express the kilogram mass, $m=\frac{\hbar}{\bar{\lambda}} \frac{1}{c}$ is naturally simply the Compton wavelength formula solved with respect to $m$, but even if this is very easy to do, it has not, to our knowledge, actually been done before in these papers. 
proof that the de Broglie hypothesis was rooted in reality. Next, in a series of steps, an entire quantum wave theory emerged from this line of thought, based on the important work of Heisenberg [32], Schrödinger [33], Klein Gordon, Pauli, and Dirac, among others. Further, the quantum mechanical theory fit experiments extremely well. And just before this development, gravity theory had evolved into Einstein's [34] general relativity theory. Since then, for more than 100 years, many of the world's most brilliant physicists have tried to unify gravity with quantum mechanics into a quantum gravity theory but without much success.

However, in our rest-mass formula, $m=\frac{\hbar}{\bar{\lambda}} \frac{1}{c}$ we do not have the de Broglie wavelength, but the reduced Compton wavelength; $\bar{\lambda}$. Compton was more of an experimental researcher than de Broglie and he had measured the wavelength of an electron around the same time that de Broglie had presented his hypothesis of the matter wave. That is, the Compton wavelength has been measured, at least indirectly. There is a very simple mathematical relation between the Compton wavelength and the de Broglie wavelength, namely $\bar{\lambda}=\bar{\lambda}_{B} \frac{v}{C}$. However, if $v=0$, then the de Broglie wavelength is infinite [35] [36], or even mathematically undefined as it is not allowed to divide by zero $\left(\lambda_{B}=\frac{\hbar}{m v \gamma}=\frac{\hbar}{m \times 0}\right)$. An infinite matter wave for a subatomic particle is, to put it mildly, a bizarre prediction. We will claim, as we have done in other papers [21], that the de Broglie wavelength is not a physical wavelength; it should be seen as a mathematical derivative of the true physical Compton wavelength. In short, the de Broglie wavelength is simply a mathematical artifact that is never needed. A theory built around the de Broglie wavelength will, in general, give a series of correct predictions, but the interpretations will often be absurd, as one has not discerned what matter is directly linked to the Compton wavelength and what is linked to the de Broglie wavelength. Why does modern physics have two different types of wavelengths for mass-one being the experimentally-observed Compton wavelength, the other being the hypothetical de Broglie wavelength? Well, this is a topic for another time.

Let's return to our mass definition in $\mathrm{kg}$ in terms of the Compton wavelength. The formula can be rewritten as:

$$
\frac{\hbar}{\bar{\lambda}} \frac{1}{c}=\frac{\frac{c}{\bar{\lambda}}}{\frac{c}{\frac{\hbar}{1 \times c}}}
$$

We can see that the $\mathrm{kg}$ of the mass in question is simply the Compton frequency of the mass in question divided by the Compton frequency of one $\mathrm{kg}$. That is, the kg definition of mass at a deeper quantum level is a frequency ratio. At each Compton time we will claim there is a Planck mass event. Such Planck 
mass events consist of two indivisible particles colliding. Such indivisible particles, when not colliding with other particles, move at the speed of light over the reduced Compton length. For example, an electron will then have the following number of Planck mass events per second:

$$
f_{e}=\frac{c}{\bar{\lambda}_{e}} \approx 7.76 \times 10^{20}
$$

Each Planck mass event is $10^{-8} \mathrm{~kg}$, but the Planck mass event only lasts for one Planck time, so this gives a mass in $\mathrm{kg}$ for the electron of:

$$
m_{e}=\frac{c}{\bar{\lambda}_{e}} \approx 7.76 \times 10^{20} \times m_{p} t_{p}=\frac{\frac{c}{\bar{\lambda}}}{\frac{c}{\frac{\hbar}{1 \times c}}}=10^{-31} \mathrm{~kg}
$$

However, this mass definition that indeed is a collision ratio does not tell anything about how long each collision lasts; it disappears in the equation, as the Planck length will cancel out between the Planck mass in terms of $\mathrm{kg}$ and the Planck time. The standard kg definition of mass is a collision ratio, and that is all we need when working with most observable phenomena. An exception to this is gravity. Gravity is not some magical force; all mass is also gravity. That is, gravity is the collisions between the indivisible particles that exist in matter. The collision only lasts for a Planck time, as we can find from gravity observations. This is, however, not embedded in today's mass definition, and it must come from somewhere in the gravity models to make the gravity formulas predict correctly. This is where the gravity constant comes in. The so-called Newton's gravitational constant adds to the formula what is missing in the kg definition of mass. Luckily what is missing is only something that is constant, namely the Planck length, and we need to take something out of the kilogram mass, namely the Planck constant. The Planck constant is the units of energy relative to the collision ratio in a kg. That is, the Planck constant is the amount of energy in an indivisible particle in the form of a collision ratio where the collision ratio is relative to the collisions in one kg per second.

The quantum aspects of this theory and a unified quantum gravity theory are explained in much more detail in [21] [22] [37] [38]. Just as important is the fact that one can find the Planck length (and other Planck units such as the Planck time and the Planck mass) totally independently of any knowledge of $G$, see [39] [40] [41]. The Newton gravitational constant that Newton never invented or used is, at a deeper level, a composite constant of the form $G=\frac{l_{p}^{2} c^{3}}{\hbar}$ as described by Haug in some of the papers just mentioned, as well as in [25] [27] [42], something we soon will get back to in this paper.

\section{The Newton Mass from a Quantum Perspective, the True Mass and the Newton God Particle}

Let us look closer at what the "mysterious" mass we get out of the original New- 
ton formula actually represents from a quantum particle perspective. The reason we use the term "God Particle" is simply because Newton called such particles so:

“... and that these primitive Particles being Solids, are incomparably harder than any porous Bodies compounded of them; even so very hard, as never to wear or break in pieces, no ordinary Power being able to divide what God himself made one in the first Creation." Isaac Newton, see full quote in the appendix.

With this, we think Newton indicated that the indivisible particle was the most fundamental of all particles. We will next show how we can measure important properties of this particle that we now have reasons to think are directly linked to the Planck scale.

The mass of the Earth, for example, we predicted (using Newton's original formula) to be $1.52 \times 10^{-11}$ Light-seconds. We believe that we can find the mass of Newton's indivisible particle from this and claim it must be given by the following formula (a formula we have already shown is directly linked to the Planck length, [43])

$$
\tilde{m}_{i}=\sqrt{\tilde{M} \bar{\lambda}}
$$

where $\bar{\lambda}$ is the reduced Compton wavelength of the Newtonian mass $\tilde{M}$ of for example the Earth, (e.g. the gravity object of which we have observed the mass). How can we find the Compton wavelength of the Earth? We can measure the Compton wavelength of an electron without knowing the mass of the electron. The reduced Compton wavelength of an electron can be found by Compton scattering and it is about $3.86 \times 10^{-13} \mathrm{~m}$. Also be aware that the Planck constant is not needed for finding this, because we have:

$$
\lambda_{e}=\frac{\lambda_{\gamma, 2}-\lambda_{\gamma, 1}}{1-\cos \theta}
$$

where $\lambda_{\gamma, 1}$ and $\lambda_{\gamma, 2}$ are the wavelength of the photon before and after it hit the electron, and $\theta$ is the angle between the incoming and outgoing photon. In light-seconds, the reduced Compton wavelength $\left(\bar{\lambda}_{e}=\lambda_{e} /(2 \pi)\right)$ of the electron is about $1.28 \times 10^{-21}$ light-seconds. This can be measured without knowing the mass of the electron first, see also [44]. Further, the Compton wavelength of a proto can be found by simply checking the cyclotron frequency of a proton relative to an electron. The cyclotron frequency is given by:

$$
f=\frac{q B}{2 \pi m}
$$

where $q$ is the charge of the particle, and $B$ is the magnetic field, and $m$ is the mass of the particle. Since protons and electrons have the same charge, we must have:

$$
\frac{f_{e}}{f_{p}}=\frac{\frac{q B}{2 \pi m_{e}}}{\frac{q B}{2 \pi m_{P}}}=\frac{m_{P}}{m_{e}}=\frac{\bar{\lambda}_{e}}{\bar{\lambda}_{P}} \approx 1836.15
$$


The well-known (measured) cyclotron frequency ratio [45] [46] is about 1836.15247, so the reduced Compton wavelength of the proton is simply the measured reduced Compton wavelength of the electron divided by the cyclotron frequency ratio, that is $\bar{\lambda}_{P}=\bar{\lambda}_{e} \frac{f_{e}}{f_{P}} \approx \frac{\bar{\lambda}_{e}}{1836.15247}$. Interest in the Compton wavelength of the proton goes back to at least 1958 and has recently garnered more interest; see [47] [48]. Now we just need to know the number of protons (assuming neutrons have same mass or do we need to make a slight adjustment for this) in the Earth, which we could count hypothetically, even if this is impossible directly in practice, but we will soon look at indirect methods to do so. In any case, there are about $3.57 \times 10^{51}$ protons in the Earth (we assume neutrons have approximately the same mass as protons). In addition, there would be a small adjustment for binding energy, the nuclear binding energy, and the bond energy that keeps atoms together, but that is so small compared to the rest-mass energy of the atoms that it will not make much of a difference in the predicted Compton wavelength. The reduced Compton length of the Earth is then given by:

$$
\begin{aligned}
\bar{\lambda}_{E} & =\sum_{i=1}^{n}=\frac{1}{\frac{1}{\bar{\lambda}_{1}}+\frac{1}{\bar{\lambda}_{2}}+\frac{1}{\bar{\lambda}_{3}}+\cdots+\frac{1}{\bar{\lambda}_{n}}}=\frac{1}{3.57 \times 10^{51} \times \frac{1}{\bar{\lambda}_{e} / 1836.15}} \\
& =1.96 \times 10^{-76} \text { light-seconds }
\end{aligned}
$$

The mass of Newton's indivisible particle we can now calculate by:

$$
\tilde{m}_{i}=\sqrt{\tilde{M} \bar{\lambda}}=\sqrt{1.52 \times 10^{-11} \times 1.96 \times 10^{-76}} \approx 5.46 \times 10^{-44} \text { light-seconds }
$$

Some will recognize this number; it is the Planck time, which is $5.46 \times 10^{-44}$ seconds. This is the case because we have chosen seconds as our time scale but remember this is also directly linked to our length scale. The ultimate subatomic mass is a collision between two indivisible particles; this collision lasts for approximately $5.46 \times 10^{-44}$ seconds. Our interpretation is that two indivisible particles spend this amount of time in collision (standing still) during the period in which one non-colliding indivisible particle (moving at the speed of light) travels a distance equal to the Planck length, that has a distance of $5.46 \times 10^{-44}$ light seconds (or approximately $1.61 \times 10^{-35} \mathrm{~m}$ ). This is explained in more detail, but from a slightly different perspective, in our two collision space-time unified quantum gravity papers, see [21] [38].

Keep in mind, we never relied on the so-called Newton gravitational constant (that Newton never invented) that was invented to fit the arbitrary kilogram mass, and the mass definition of $\mathrm{kg}$ (pounds); as we have said, at a deeper level the kilogram mass is just a collision ratio. Nor do we need the Planck constant to find the Planck time [49]. One can mistakenly think this is only theory as it seems impossible to directly count the number of protons in the Earth. Still, we can do this indirectly. This is when a Cavendish apparatus comes in handy. Here, we can start out by finding the Newton gravitational mass of a small practical 
mass like a lead ball, given by:

$$
\tilde{M}_{c}=\frac{2 \pi^{2} R^{2} L \theta}{T^{2}}
$$

where $\tilde{M}_{c}$ is the Newton mass of one of the large balls in the Cavendish apparatus and $L$ is the distance between the smallest balls in the Cavendish apparatus, and $R$ is the distance from the centre of the small ball to the centre of the larger ball, and $\theta$ is the angle of deflection (in radians), and $T$ is the oscillation time. This formula is only valid when $c=1$; otherwise one must divide it by $c^{3}$.

To find the Compton wavelength of the ball in the Cavendish apparatus, we can count the number of protons in that object; this is also a challenge, but is fully possible; see [50] [51] [52]. When we know the Newton mass (light-seconds) of the ball, we can easily find the Newton mass of the Earth relative to that. Also, if we know the Compton wavelength of the mass in the Cavendish apparatus (by counting atoms in it as described above), then we can find the reduced Compton wavelength of the Earth from the following equation:

$$
\bar{\lambda}_{E}=\frac{\tilde{M}_{c}}{\tilde{M}_{E}} \bar{\lambda}
$$

where $\bar{\lambda}$ is the reduced Compton wavelength of the sphere in the Cavendish apparatus. We could also have found the reduced Compton wavelength of the Earth simply by using the Compton formula:

$$
\bar{\lambda}_{E}=\frac{\hbar}{M_{E} c}
$$

but then we need to know the Planck constant, and part of our purpose is to demonstrate we need fewer constants than in standard physics when understanding gravity and physics from a deeper perspective.

We also have that:

$$
\tilde{M}=\frac{G}{c^{3}} M=\frac{l p^{2} c^{3}}{\hbar c^{3}} \times \frac{\hbar}{\bar{\lambda}} \frac{1}{c}=\frac{l_{p}}{c} \frac{l_{p}}{\bar{\lambda}}
$$

which is the collision time of that mass over the shortest possible time interval it can be observed, as described by Haug in his unified quantum gravity theory [53]. Again, the collisions between indivisible particles last only for the Planck time; this is given by $t_{p}=\frac{l_{p}}{c}$, and multiplied by how often these collisions happen $\frac{l_{p}}{\bar{\lambda}}$. The part $\frac{l_{p}}{\bar{\lambda}}$ can also be seen as a frequency probability if $l_{p}<\bar{\lambda}$, when observed over the shortest possible time interval, which is the Planck time. Be aware that for anything that has been measured in relation to the Newton formula, one of the masses in the derivations for what one wants to predict will always cancel out; we are always operating with just $G M$ in any observable prediction and never $G M m$. Modern physics appears to have missed the point that the invented $G M$ is actually identical to the mass in the original Newton formula. That $G M$ is the Newtonian mass holds when we have linked length and time 
through the speed of light, e.g., when $c=1$. When we have units such that $c<>1$, then the collision time mass is given by $\tilde{M}=\frac{l_{p}}{c} \frac{l_{p}}{\bar{\lambda}}=\frac{G M}{c^{3}}=\frac{1}{2} \frac{r_{s}}{c}$. In the special case $c=1$ we naturally get $\tilde{M}=\frac{1}{2} r_{s}$. When $c<>1$ it is interesting to note that we also have ${ }^{4} l_{p}=\sqrt{\frac{1}{2} r_{s} \bar{\lambda}}$.

Back to the gravity constant $G$; why on earth would the universe invent something that is length cubed divided by time and $\mathrm{kg}$ (the output units of $G$ ). Of course, the universe never invented such a thing. Modern physics invented a gravity constant to fit a misinterpreted mass view of Newton's formula, which was needed to get physicists' ill-specified mass model to fit experiments. Newton never mentioned a gravitational constant himself. He calculated relative masses based on orbital time squared (and adjusted for distance between the gravity objects; that is, the masses.).

\section{The So-Called Newton's Gravity Constant $G$ Is Just a Composite Constant Needed to Fix the Incomplete Kilogram Mass}

In 1984, Cahill [54] already suggested that the Newtonian gravity constant could perhaps be a composite constant of the form $G=\frac{\hbar c}{m_{p}^{2}}$ and that the Planck units could be more fundamental; in other words, simply solving the Planck mass formula $m_{p}=\sqrt{\frac{\hbar c}{G}}$ with respect to $G$. However, in 1987, Cohen [55] pointed out that if one needs $G$ to find the Planck units this will simply lead to a circular problem, so it seemed one needed to know $G$. This is the main view among most researchers to this day, and has been repeated as late as 2016 in an interesting paper by McCulloch [56]. However, in recent years, we have had a breakthrough in understanding the Planck units. We can now extract the Planck length and Planck time from a series of gravity observations without any prior knowledge of $G$, $\mathcal{c}$, and $\hbar$, see [40] [41] [57]. There also exist other suggestions for how to get the gravity constant from such things as its hypothetical relation to electromagnetic constants to suggestions of how to extract $G$ from cosmological constants; see, for example, [58] [59] [60]. However, here we will focus on expressing the gravity constant from the Planck units as this seems to lead to a significant step forward in understanding gravity. Some of these approaches are actually closely related when they are compared carefully; see [61]. In 2016 we [42] suggested this to express $G$ as a composite constant of the form:

$$
G=\frac{l_{p}^{2} c^{3}}{\hbar}
$$

This is nothing more than solving the Planck length formula of Max Planck

\footnotetext{
${ }^{4}$ As first described by [43].
} 
with respect to $G$. Back then, I had also not been able to yet solve the circular problem. That is, we had not yet found a way to find $l_{p}$ or other Planck units independent of $G$. A year later, Haug [39] solved the circular problem for the first time, so $G$ can indeed be expressed as a composite constant and the Planck length can be found independent of any knowledge of $G$. Later on, we showed $l_{p}$ can be found independent of any prior knowledge of $G, c$ and $\hbar$. See, for example, [62].

Still, it is first when one combines this composite view of $G$ with the idea that any kilogram mass can be expressed as $m=\frac{\hbar}{\bar{\lambda}} \frac{1}{c}$, one gets a real breakthrough in the understanding of gravity. All observable gravity phenomena rooted in today's standard gravity theory contain $G M$ and not $G M m$. The 1873, modified, Newton gravity formula indeed contains $G M m$, but the small mass $m$ always cancels out in derivations of formulas that can be used to predict observable gravity phenomena, and can thereby be checked with observations. This is for observable gravity phenomena where the small mass $m$ has insignificant gravitational impact relative to $M$; in other words, when we have $m \ll M$. For real two body problems where both masses are significantly large relative to each other to have significant impact the gravity parameter is $\mu=G\left(M_{1}+M_{2}\right)=G M_{1}+G M_{2}$, so then one multiplies both the kilogram masses with the gravity constant.

One can ask why it is necessary to always multiply the mass with $G$ when used it for gravity. At a superficial level, this is simply how we have to calibrate the gravity formula for it to be useful for predictions. First, we must find the value of $G$ from one gravity observational phenomena and then we can use the same $G$ to predict other types of things related to gravity that we can observe. In other words, $G$ seems to be a constant; it is an empirically-observed or calibrated constant, not a derived constant, or something understood from a very deep perspective. The physics' community has no idea what $G$ truly represents, or exactly why it is there. In 1961, Thüring [63] concluded that $G$ had been inserted quite ad hoc and that it is not clear how it is related to the physical nature. In our view, $G$ contains something missing in the model. When one introduced the kilogram mass, something was missing in the formula $F=M m / R^{2}$ so one had to multiply it by an unknown constant and get $F=G M m / R^{2}$. The constant $G$ was unknown and had to be found by calibration to observable data. It then worked, but no one knew exactly why, because they had, and still have, no knowledge of why exactly $G$ must be included and what it represents at a deeper level. This we can first really understand when we multiply $G$ in the composite form, $G=\frac{l_{p}^{2} c^{3}}{\hbar}$, with the kilogram mass. This gives:

$$
G M=\frac{l_{p}^{2} c^{3}}{\hbar} \times \frac{\hbar}{\bar{\lambda}} \frac{1}{c}=c^{3} \frac{l_{p}}{c} \frac{l_{p}}{\bar{\lambda}}
$$

That is, the Planck constant in the kilogram mass cancels out with the Planck constant embedded in $G$, so to calculate $G M$ we need less information than to 
find $G$ and $M$ separately. In our view, $G$ is needed to get $\hbar$ out of the kilogram mass and $l_{p}^{2}$ into the mass. Further:

$G M=c^{3} \frac{l_{p}}{c} \frac{l_{p}}{\bar{\lambda}}$ can be seen as a gravity constant $c^{3}$, multiplied by a new mass definition $\bar{m}=\frac{l_{p}}{c} \frac{l_{p}}{\bar{\lambda}}$, which we have called collision-time mass.

This mean the Newton gravity force formula can be described as:

$$
F=c^{3} \frac{\tilde{M} \tilde{m}}{R^{2}}
$$

This force formula does not give the same output units as the 1873 version of the Newton formula, as its output unit is $\mathrm{m} \cdot \mathrm{s}^{-1}$ versus the 1873 formula's output that gives $\mathrm{m} \cdot \mathrm{kg} \cdot \mathrm{s}^{-2}$, so one could mistakenly think there must therefore be something very wrong with our newly-suggested gravity force formula, see also [41]. The thing is that the Newton's gravity force is never observed, and neither is the force coming from the 1873 formula. What is observable is when the small $\mathrm{m}$ has canceled out from the formula through derivations of predictions of observable gravitational phenomena. The new gravity force formula is simple and give exactly the same predictions and also the same output units as the as the 1873 formula, and in the special case of setting $c=1$, the new formula is the original Newton formula: $F=\frac{\tilde{M} \tilde{m}}{R^{2}}$.

Table 1 shows the original Newton formula as well as observations we can derive from it, in addition to the modified Newton version of 1873 , which has the a gravity constant $G$. The two formulas, at a deeper level, predict exactly the same for observable phenomena. However, the Newton formula is simpler, requires fewer constants and is much more intuitive. If two theories are identical in predictions, then the simplest theory should win. In the original Newton formulation, we are totally independent of the value of $\hbar$, so this is not simply setting $G=c=\hbar=1$, in the original Newton formula $F=\frac{\tilde{M} \tilde{m}}{R^{2}}$ all that is set to 1 is $c$.

\section{The Uncertainty in Measurement of $G$ Is It Still Relevant}

We have demonstrated in this paper that Newton never invented nor used the so-called Newton gravitational constant $G$. Further, from Table 1, it is clear $G$ is not needed to predict any observable gravitational phenomena. The Newtonian gravitational constant introduced in 1873 is needed when one uses the incomplete kilogram definition of mass, to fix that kilogram mass into a gravitational mass. The kilogram mass is not in line with Newton's thought that matter ultimately consists of indivisible particles, which recent research strongly indicates are linked to the Planck length and Planck time.

It is well known that there is a large uncertainty in the measurement of the Newtonian gravitational constant compared to most other physical constants. See, for example [64]-[69]. However, it would be a misunderstanding to think 
Table 1. In the first formula column, the table shows what is rooted in the 1873 modified Newton theory, and the second formula column shows the original Newton formula. In addition, we show what both the 1873 framework and the original Newton framework means at the deepest level, where both theories are identical, except in the original Newton theory $c=1$. Further, pay attention to the fact that all observable gravity phenomena are linked to $G M$ and not $G M m$ in the 1873 modified Newton gravity theory and only to $\tilde{M}$ in the original Newton theory, rather than $\tilde{M} \tilde{m}$.

Non observable (contains $G M m$ or $\tilde{M} \tilde{m}$ )

1873 modified Newton and forward:

“Original” Newton:

Gravity force

$$
F=G \frac{M m}{R^{2}}\left(\mathrm{~kg} \cdot \mathrm{m} \cdot \mathrm{s}^{-2}\right)
$$$$
F=c^{3} \frac{\tilde{M} \tilde{m}}{R^{2}}=\frac{\tilde{M} \tilde{m}}{R^{2}}\left(\mathrm{~m} \cdot \mathrm{s}^{-1}\right) \text { when } c=1
$$

Mass must be

$$
M=\frac{\hbar}{\bar{\lambda}_{M}} \frac{1}{c}(\mathrm{~kg})
$$

Gravitational constant

$$
G,\left(G=\frac{l_{p}^{2} c^{3}}{\hbar}\right)
$$$$
C=1
$$

Observable predictions, identical for the two methods: (contains only $G M$ )

Gravity acceleration

Orbital velocity

Orbital time

Velocity ball Newton cradle

Periodicity Pendulum (clock)

Frequency Newton spring

Gravitational red-shift

Time dilation

Gravitational deflection (GR)

$$
g=\frac{G M}{R^{2}}=\frac{c^{2}}{R^{2}} \frac{l_{p}^{2}}{\bar{\lambda}_{M}}
$$$$
g=\frac{\tilde{M}}{R^{2}}=\frac{1}{R^{2}} \frac{l_{p}^{2}}{\bar{\lambda}_{M}}
$$

$$
v_{o}=\sqrt{\frac{G M}{R}}=c l_{p} \sqrt{\frac{1}{R \bar{\lambda}_{M}}}
$$$$
v_{o}=\sqrt{\frac{\tilde{M}}{R}}=l_{p} \sqrt{\frac{l_{p}^{2}}{R \bar{\lambda}_{M}}}
$$$$
T=\frac{2 \pi R}{\sqrt{\frac{G M}{R}}}=\frac{2 \pi \sqrt{\bar{\lambda} R^{3}}}{c l_{p}}
$$

$$
T=\frac{2 \pi R}{\sqrt{\frac{\tilde{M}}{R}}}=\frac{2 \pi \sqrt{\bar{\lambda} R^{3}}}{l_{p}}
$$

$$
v_{\text {out }}=\sqrt{2 \frac{G M}{R^{2}} H}=\frac{c l_{p}}{R} \sqrt{\frac{H}{\bar{\lambda}}}
$$

$$
v_{\text {out }}=\sqrt{2 \frac{\tilde{M}}{R^{2}} H}=\frac{l_{p}}{R} \sqrt{\frac{H}{\bar{\lambda}}}
$$

$$
T=2 \pi \sqrt{\frac{L}{g}}=2 \pi R \sqrt{\frac{L}{G M}}=\frac{2 \pi R}{c l_{p}} \sqrt{L \bar{\lambda}}
$$$$
T=2 \pi \sqrt{\frac{L}{g}}=T=2 \pi R \sqrt{\frac{L}{\tilde{M}}}=\frac{2 \pi R}{l_{p}} \sqrt{L \bar{\lambda}}
$$

$f=\frac{1}{2 \pi} \sqrt{\frac{k}{m}}=\frac{1}{2 \pi R} \sqrt{\frac{G M}{x}}=\frac{c l_{p}}{2 \pi R} \sqrt{\frac{1}{\bar{\lambda} x}}$

$$
f=\frac{1}{2 \pi} \sqrt{\frac{k}{\tilde{m}}}=\frac{1}{2 \pi R} \sqrt{\frac{\tilde{M}}{x}}=\frac{l_{p}}{2 \pi R} \sqrt{\frac{1}{\bar{\lambda} x}}
$$

$$
z=\frac{\sqrt{1-\frac{2 G M}{R_{1} c^{2}}}}{\sqrt{1-\frac{2 G M}{R_{2} c^{2}}}}-1=\frac{\sqrt{1-\frac{2 l_{p}^{2}}{R_{1} \bar{\lambda}_{M}}}}{\sqrt{1-\frac{2 l_{p}^{2}}{R_{2} \bar{\lambda}_{M}}}}-1
$$

Observable predictions (from GR): (contains only $G M$ or only $\tilde{M}$ )

$$
\begin{gathered}
T_{R}=T_{f} \sqrt{1-\sqrt{\frac{2 G M}{R}}^{2} / c^{2}}=T_{f} \sqrt{1-\frac{2 l_{p}^{2}}{R \bar{\lambda}_{M}}} \\
\delta=\frac{4 G M}{c^{2} R}=\frac{4}{R} \frac{l_{p}^{2}}{\bar{\lambda}_{M}}
\end{gathered}
$$$$
T_{R}=T_{f} \sqrt{1-\sqrt{\frac{2 \bar{M}^{2}}{R}} / c^{2}}=T_{f} \sqrt{1-\frac{2 l_{p}^{2}}{R \bar{\lambda}_{M}}}
$$$$
\delta=\frac{4 c^{3} \bar{M}}{c^{2} R}=\frac{4}{R} \frac{l_{p}^{2}}{\bar{\lambda}_{M}}
$$ 


\section{Continued}

Advance of perihelion

Quantum analysis:

Constants needed

Variable needed

$$
\sigma=\frac{6 \pi G M}{a\left(1-e^{2}\right) c^{2}}=\frac{6 \pi}{a\left(1-e^{2}\right)} \frac{l_{p}^{2}}{\bar{\lambda}_{M}}
$$$$
\sigma=\frac{6 \pi \tilde{M}}{a\left(1-e^{2}\right) c^{2}}=\frac{6 \pi}{a\left(1-e^{2}\right)} \frac{l_{p}^{2}}{\bar{\lambda}_{M}}
$$

$G, \hbar$, and $c$ or $l_{p}, \hbar$, and $c$

$l_{p}$ and indirectly $c$, but $c=1$

one for mass size

we are getting away from this uncertainty after we have got rid of $G$. So, this paper is not about improving or getting rid of this uncertainty. From a deeper perspective, the uncertainty in $G$ ultimately comes from uncertainty in measurements of the Planck length. This also explains why the uncertainty in $G$ is so large compared to in what has been found in most other physical constants. The reason is that the Planck length is the shortest possible observable length, and it is therefore not so strange that it is hard to measure it accurately when it is the smallest of all things there are.

The standard uncertainty in the gravity constant $G$ is exactly twice that of the standard uncertainty in the Planck length. Just as an illustrative example, assume the measured standard uncertainty in the Planck length is $1 \%$, then relative uncertainty in the gravitational constant must be:

$$
\frac{\partial G}{\partial l_{p}} \frac{\frac{l_{p}}{100}}{G}=2 \%
$$

NIST 2018 CODATA states the one standard deviation uncertainty in the gravity constant is given by $2.2 \times 10^{-5}$, and for the Planck length the one standard deviation uncertainty is given as $1.1 \times 10^{-5}$. This perfectly matches our view that the standard uncertainty in the Newton gravity constant is exactly twice of that of the Planck length. But since where we have $G$ in the 1873 Newtonian framework we have $l_{p}^{2}$ embedded, this since $G=\frac{l_{p}^{2} c^{3}}{\hbar}$ as understood from a deeper level, then the standard uncertainty in the gravity observations are the same as before. Bear in mind that $c$ and $\hbar$ are defined as exact constants so they do not add to any uncertainty in $G$ or in gravitational observations, because $\hbar$ also cancels out for any observable gravity phenomena. All these studies, which try as accurately as possible to find the value of the gravitational constant, can be seen as simply methods to find an accurate value of the Planck length, even if the researchers looking into measuring $G$ are not aware of this. They are of the view that the Planck length only can be found after one has found $G, c$ and $h$ through dimensional analysis. In recent years, we have demonstrated how to find the Planck length and Planck time independent on any knowledge of $G$ and $h$ and even of $c$. Still, these experiments, trying to accurately measure $G$, are just as relevant as before, but it is the uncertainty in $l_{p}^{2}$ the experimenters are looking at, 
without knowing so. It could be that when this becomes widely known, one could devise even more accurate ways to measure $l_{p}^{2}$, but this only time can tell. We do not claim to know any new ways to measure $G$ more accurately than before.

From Table 1, we see that both the standard 1873 Newtonian formalization, as well as the original Newtonian formulation that is without $G$, when understood from a deeper perspective, contain the same two constants for prediction of all observable phenomena; that is, $l_{p}$ and $c$.

Still, our insight that we do not need $G$ to make gravity predictions is not an argument to reduce the uncertainty in gravity measurements. It is an argument for the possibility to understand gravity through deeper and simpler principles. It is also an argument to reduce the number of universal constants from $G, h$ and $c$ to just $l_{p}$ and $c$.

\section{Conclusion}

As we have seen, it is by using Newton's original formula that we obtain the correct unit measure of mass. The kg definition of mass is a manmade, arbitrary unit of mass that has caused great confusion in modern physics. The kg definition and similar manmade arbitrary units (such as the pound) are why the gravitational constant had to be invented. Nature does not work in kg; it has its own, more fundamental units. Arbitrary incomplete units have added an unnecessary layer of complexity to modern physics, and Newton's original theory is superior in many ways. Naturally, the theory was not complete in terms of quantum mechanics and relativity theory. However, if the field of physics had stayed with Newton's original formula, it is possible that a full understanding of mass and a unified quantum theory might have been developed much earlier.

\section{Acknowledgements}

Thanks to two anonymous reviewers as well as to Balanced View (on twitter) for useful comments.

\section{Conflicts of Interest}

The author declares no conflicts of interest regarding the publication of this paper.

\section{References}

[1] Newton, I. (1686) Philosophiae Naturalis Principia Mathematica. Joseph Streater for the Royal Society, London. https://doi.org/10.5479/sil.52126.39088015628399

[2] Simonyi, K. (2010) The Cultural History of Physics. CRC Press, Boca Raton.

[3] Milstrom, J. (2018) The Hunt for Earth Gravity. Springer, Berlin.

[4] Cohen, I.B. (1998) Archive for History of Exact Sciences, 53, 83-95. https://doi.org/10.1007/s004070050022

[5] Chalmers, A. (2009) Newton's Atomism and Its Fate. In: Chalmers, A., Ed., The Scientist's Atom and the Philosopher's Stone, Volume 279 of the Series Boston Studies in the Philosophy of Science, Springer, Berlin, 123-138.

https://doi.org/10.1007/978-90-481-2362-9 7 
[6] Schofield, R.E. (1981) American Journal of Physics, 49, 211-216. https://doi.org/10.1119/1.12519

[7] Newton, I. (1704) Opticks. Smith \& Walford, London.

[8] Cavendish, H. (1798) Philosophical Transactions of the Royal Society of London, (Part II), 88, 469-526. https://doi.org/10.1098/rstl.1798.0022

[9] Michell, J. (1784) Philosophical Transactions of the Royal Society, 74. https://doi.org/10.1098/rstl.1784.0008

[10] Cornu, A. and Baille, J.B. (1873) Comptes Rendus de P Académie des Sciences Paris, 76, 954-955.

[11] Boys, C.V. (1894) Nature, 50, 571. https://doi.org/10.1038/050571a0

[12] Planck, M. (1899) Natuerliche Masseinheiten. Der Königlich Preussischen Akademie Der Wissenschaften.

[13] Planck, M. (1906) Vorlesungen über die Theorie der Wärmestrahlung. J.A. Barth, Leipzig, 163. See Also the English Translation “The Theory of Radiation” (1959) Dover.

[14] Planck, M. (1928) Einführung in die allgemeine Mechanik. Verlag von Hirtzel, Leipzig.

[15] Langacker, P. (2017) Can the Laws of Physics Be Unified? Princeton University Press, Princeton. https://doi.org/10.1515/9781400885503

[16] Haug, E.G. (2021) Physics Essays, 340, 502. https://doi.org/10.4006/0836-1398-34.4.502

[17] Adler, C.G. (1987) American Journal of Physics, 55, 739-743. https://doi.org/10.1119/1.15314

[18] Hecht, E. (2009) The Physics Teacher, 47, 336-341. https://doi.org/10.1119/1.3204111

[19] Leibniz, G.W. (1989) A Brief Demonstration of a Notable Error of Descartes and Others Concerning a Natural Law? In: Loemker, L.E., Ed., Philosophical Papers and Letters, the New Synthase Historical Library (Texts and Studies in the History of Philosophy), Vol. 2, Springer, Dordrecht, 1686.

[20] Jacob Gravesande, W. (1720) Mathematical Elements of Physicks, prov'd by Experiments: Being an Introduction to Sir Isaac Newton's Philosophy. Made English, and Illustrated with 33 Copper-Plates, Revis'd and Corrected, by Dr. John Keill. London.

[21] Haug, E.G. (2020) Physics Essays, 330, 46. https://doi.org/10.4006/0836-1398-33.1.46

[22] Haug, E.G. (2022) Unified Quantum Gravity Field Equation Describing the Universe from the Smallest to the Cosmological Scales. Physics Essays, Accepted for Publication and Forthcoming in Vol. 35.

[23] Augousti, A.T. and Radosz, A. (2006) European Journal of Physics, 27, 331-335. https://doi.org/10.1088/0143-0807/27/2/015

[24] Compton, A.H. (1923) Physical Review, 210, 483-502. https://doi.org/10.1103/PhysRev.21.483

[25] Haug, E.G. (2016) International Journal of Astronomy and Astrophysics, 6, 206-217. https://doi.org/10.4236/ijaa.2016.62017

[26] Haug, E.G. (2016) A Note on the Dimensionless Gravitational Coupling Constant. https://vixra.org/pdf/1604.0198v1.pdf

[27] Haug, E.G. (2018) International Journal of Astronomy and Astrophysics, 8, 6-23. https://doi.org/10.4236/ijaa.2018.81002 
[28] Broglie, L. (1930) An Introduction to the Study of Wave Mechanics. Metheum \& Co., Essex.

[29] Broglie, L. (1924) Recherches sur la théorie des quanta. PhD Thesis, Masson, Paris.

[30] Davisson, C. and Germer, L.H. (1927) Physical Review, 30, 705-740. https://doi.org/10.1103/PhysRev.30.705

[31] Thomson, G.P. and Reid, A. (1927) Nature, 119, 890. https://doi.org/10.1038/119890a0

[32] Heisenberg, W. (1927) Zeitschrift für Physik, 43, 172-198. https://doi.org/10.1007/BF01397280

[33] Schrödinger, E. (1926) Physical Review, 28, 1049-1070. https://doi.org/10.1103/PhysRev.28.1049

[34] Einstein, A. (1916) Näherungsweise integration der feldgleichungen der gravitation. Sitzungsberichte der Königlich Preussischen Akademie der Wissenschaften, Berlin.

[35] Chauhan, H., Rawal, S. and Sinha, R.K. (2011) Wave-Particle Duality Revitalized: Consequences, Applications and Relativistic Quantum Mechanics. https://arxiv.org/pdf/1110.4263.pdf

[36] Lvovsky, A.I. (2018) Quantum Physics: An Introduction Based on Photons. Springer, Berlin.

[37] Haug, E.G. (2014) Unified Revolution, New Fundamental Physics. E.G.H. Publishing, Oslo.

[38] Haug, E.G. (2020) Rethinking the Foundation of Physics and Its Relation to Quantum Gravity and Quantum Probabilities: Unification of Gravity and Quantum Mechanics. https://www.preprints.org/manuscript/202012.0483/v2 https://doi.org/10.20944/preprints202012.0483.v1

[39] Haug, E.G. (2017) Applied Physics Research, 9, 58. https://doi.org/10.5539/apr.v9n6p58

[40] Haug, E.G. (2020) Journal Physics Communication, 4, Article ID: 075001. https://doi.org/10.1088/2399-6528/ab9dd7

[41] Haug, E.G. (2021) Journal of Physics Communication, 5, Article ID: 025005. https://doi.org/10.1088/2399-6528/abe4c8

[42] Haug, E.G. (2016) Physics Essays, 29, 558-561. https://doi.org/10.4006/0836-1398-29.4.558

[43] Haug, E.G. (2018) Preprints, 2018, Article ID: 2018090396. https://www.preprints.org/manuscript/201809.0396/v1

[44] Prasannakumar, S., Krishnaveni, S. and Umesh, T.K. (2012) European Journal of Physics, 33, 65-72. https://doi.org/10.1088/0143-0807/33/1/005

[45] Gräff, G., Kalinowsky, H. and Traut, J. (2014) Zeitschrift für Physik A Atoms and Nuclei, 297, 35-39. https://link.springer.com/article/10.1007/BF01414243 https://doi.org/10.1007/BF01414243

[46] Van-Dyck, R.S., Moore, F.L., Farnham, D.L. and Schwinberg, P.B. (1985) International Journal of Mass Spectrometry and Ion Processes, 660, 327. https://doi.org/10.1016/0168-1176(85)80006-9

[47] Levitt, L.S. (1958) Experientia, 14, 233. https://doi.org/10.1007/BF02159173

[48] Trinhammer, O.L. and Bohr, H.G. (2019) EPL, 128, 21001. https://doi.org/10.1209/0295-5075/128/21001

[49] Haug, E.G. (2021) Journal of Applied Mathematics and Physics, 9, 1076-1088. https://doi.org/10.4236/jamp.2021.95074 
[50] Bettin, H., Fujii, K., Man, J., Mana, G., Massa, E. and Picard, A. (2013) Annalen der Physik, 525, 680-687. https://doi.org/10.1002/andp.201300038

[51] Shi-Song, L., et al. (2014) Chinese Physics B, 24, Article ID: 010601. https://doi.org/10.1088/1674-1056/24/1/010601

[52] Massam, E. and Mana, G. (2016) Counting atoms. Nature Physics, 12, 522. https://doi.org/10.1038/nphys3754

[53] Haug, E.G. (2021) Quantum Gravity Hidden in Newton Gravity and How to Unify It with Quantum Mechanics. In: Krasnoholovets, V., Ed., The Origin of Gravity from the First Principles, NOVA Publishing, New York, 133-216.

[54] Cahill, K. (1984) Lettere al Nuovo Cimento, 39, 181-184. https://doi.org/10.1007/BF02790586

[55] Cohen, E.R. (1987) Fundamental Physical Constants. In: Sabbata, V. and Melniko, V.N., Eds., Gravitational Measurements, Metrology and Constants, Kluwer Academic Publishers, Amsterdam.

[56] McCulloch, M.E. (2016) Europhysics Letters (EPL), 115, 69001. https://doi.org/10.1209/0295-5075/115/69001

[57] Haug, E.G. (2021) European Journal of Applied Physics, 3, 15-20. https://www.ej-physics.org/index.php/ejphysics/article/view/133 https://doi.org/10.24018/ejphysics.2021.3.6.133

[58] Bleksley, A.E.H. (1951) South African Journal of Science, 48, 20-25. https://hdl.handle.net/10520/AJA00382353 3467

[59] Sánchez, J. (2017) Journal of High Energy Physics, Gravitation and Cosmology, 3, 87-95. https://doi.org/10.4236/jhepgc.2017.31012

[60] Kalinski, M. (2021) Journal of High Energy Physics, Gravitation and Cosmology, 7, 595-601. https://doi.org/10.4236/jhepgc.2021.72034

[61] Haug, E.G. (2022) Progress on Composite View of Newtonian Gravitational Constant and Its Link to the Planck Scale. Under Review.

[62] Haug, E.G. (2022) European Journal of Applied Physics, 4, 11-18. https://www.ej-physics.org/index.php/ejphysics/article/view/144 https://doi.org/10.24018/ejphysics.2022.4.1.144

[63] Thüring, B. (1961) Annales Academiæ Scientiarum Fennicæ A, 3, 269.

[64] Gillies, G.T. (1997) Reports on Progress in Physics, 60, 151-225. https://doi.org/10.1088/0034-4885/60/2/001

[65] Bisnovatyi-Kogan, G.S. (2006) International Journal of Modern Physics D, 15, 10471052. https://doi.org/10.1142/S0218271806008747

[66] Galli, S., Melchiorri, A., Smoot, G.F. and Zahn, O. (2009) Physical Review D, 80, Article ID: 023508. https://doi.org/10.1103/PhysRevD.80.023508

[67] Fixler, B., Foster, G.T., McGuirk, J.M. and Kasevich, M.A. (2007) Science, 315, 74-77. https://doi.org/10.1126/science.1135459

[68] Rosi, G., Sorrentino, F., Cacciapuoti, L., Prevedelli, M. and Tino, G.M. (2014) Nature, 510, 518-521. https://doi.org/10.1038/nature13433

[69] Luo, J. and Hu, Z. (2000) Classical and Quantum Gravity, 17, 2351-2363. https://doi.org/10.1088/0264-9381/17/12/307

[70] Redondi, P. (1989) Galileo: Heretic. Princeton University Press, Princeton. https://doi.org/10.1063/1.2810883

[71] Donato, M.P. (2019) Medicine and the Inquisition in the Early Modern World. Brill, Boston. https://doi.org/10.1163/9789004386464 


\section{Appendix: Some Quotations from Newton}

Below are some quotations from Newton on gravity

If there be several bodies consisting of equal particles whose forces are as the distances of the places from each, the force compounded of all the forces by which any corpuscle is attracted will tend to the common centre of gravity of the attracting bodies; and will be the same as if those attracting bodies, preserving their common centre of gravity, should unite there, and be formed into a globe. p 236

I say, that the whole force with which one of these spheres attracts the other will be reciprocally proportional to the square of the distance of the centres. The force with which one of these attracts the other will be still, by the former reasoning, in the same ratio of the square of the distance inversely. Cor. 3. The motive attractions, or the weights of the spheres towards one another, will be at equal distances of the centres as the attracting and attracted spheres conjunctly, that is, as the products arising from multiplying the spheres into each other. $\mathrm{p}$. 223.

Cor.2 The force of gravity towards several equal particles of any body is reciprocally as the square of the distance of the places of the particles. p. 393.

Cor.2 The force of gravity which tends to any one planet is reciprocally as the square of the distance of places of that planet's center. p. 393.

That all bodies gravitate towards every planet, and that the weights of bodies towards any the same planet, at equal distances from the centre of the planet, are proportional to the quantities of matter which they severally contain. p. 394, book 3.

If two spheres mutually gravitating each towards the other, if the matter in places on all sides round about and equidistant from the centres is similar, the weight of either sphere towards the other will be reciprocally as the square of the distance between their centres.

Wherefore the absolute force of every globe is as the quantity of matter which the globe contains, but the motive force by which every globe is attracted towards another, and which, in terrestrial bodies, we commonly call their weight, is as the content under the quantities of matter in both globes applied to the square of the distance between their centres (by Cor. IV, Prop. LXXVI), to which force the quantity of motion, by which each globe in a given time will be carried towards the other, is proportional. And the accelerative force, by which every globe according to its quantity of matter is attracted towards another, is as the quantity of matter in that other globe applied to the square of the distance between the centres of the two (by Cor. II, Prop. LXXVI): to which force, the velocity by which the attracted globe will, in a given time, be carried towards the other is proportional.

That there is a power of gravity tending to all bodies, proportional to the several quantities of matter which they contain. p. 397.

Newton only uses the word "mass" once in his book: 
The quantity of matter is the measure of the same, arising from its density and bulk conjunctly. It is this quantity that I mean hereafter everywhere under the name of body or mass.

In other words, mass is the quantity of matter.

In the Principia, Newton is also clear on the idea that the smallest particles of all bodies have spatial extension and are hard (indivisible) and can move. And he follows up with the comment, "And this is the foundation of all philosophy."

Since every particle of space is always, and every indivisible moment of duration is everywhere, certainly the Maker and Lord of all things cannot be never and nowhere. p. 505.

And thence we conclude the least particles of all bodies to be also extended, and hard and movable, and endowed with their proper vires inertia. And this is the foundation of all philosophy.

In his book Optica, Newton is even clearer that he think matter consists of fully-hard forever-lasting particles; that is, indivisible particles:

All these things being consider'd it seems probable to me, that Godin the Beginning form'd Matter in solid, massy, hard, impenetrable, movable Particles, of such Sizes and Figures, and in such Proportion to Space, as most conduce to the End for which he form'd them; and that these primitive Particles being Solids, are incomparably harder than any porous Bodies compounded of them; even so very hard, as never to wear or break in pieces, no ordinary Power being able to divide what God himself made one in the first Creation. While the Particles continue entire, they may compose bodies of one and the same Nature and Texture in all Ages, But should they wear away, or break in pieces, the Nature of Things depending on them, would be changed. Those minute rondures, swimming in space, from the stuff of the world: the solid, coloured table I write on, no, less than the thin invisible air I breathe, is constructed out of small colourless corpuscles; the world at close quarters looks like the night sky - a few dots of stuff, scattered sporadically through and empty vastness. Such is modern corpuscularianism.

There are many more references showing that Newton believed that the smallest particles were indivisible, even though he also said it would be hard to prove. This seems to be a view he held from the time of his unpublished notebook, to his published works Opticks and Principia. He wrote more about this in unpublished draft versions than he did in published versions. Keep in mind that even to talk about atomism had been forbidden in most of Europe for hundreds of years. Giordano Bruno was burnt at the stake in 1600 mainly for talking openly about atomism. As another example of the suppression and persecution taking place in that era, in 1624 the Paris Parliament decreed that a person maintaining or teaching atomism would be liable for the death penalty. Lancelot Law Whyte, who claimed to have worked with Albert Einstein on the unified field theory, noted,

The aggressive rise of physical atomism as an adequate explanation of the universe ... provoked a crusade (1660-1700) against it. 
In addition, recent research has shown that the Galileo affair may have been related to the fact that he openly talked about atomism; see [70]. For example, in the late 1680s, the Holy Office ordered local inquisitors to refuse to licence books which stated that: "substantial composites are not made by matter and form but by atoms or corpscules" [71].

In England, the climate for discussing atomism was slightly more relaxed than in continental Europe, but even Newton probably had to be careful, especially if he was considering the possibility of visiting France, for example. 